American Journal of Immunology 4 (3): 33-42, 2008

ISSN 1553-619X

(C) 2008 Science Publications

\title{
Hydrophobic, Polar and Hydrogen Bonding Based Drug-Receptor Interaction of Tetrahydroimidazobenzodiazepinones
}

\author{
V.K. Sahu, A.K.R. Khan, R.K. Singh and P.P. Singh \\ Department of Chemistry, Maharani Lal Kunwari (PG) College, \\ Balrampur, Uttar Pradesh, 271201, India
}

\begin{abstract}
Anti-HIV drug discovery has been increasingly focusing on HIV-1-RT (reverse transcriptase) as a potential therapeutic target. Tetrahydroimidazobenzodiazepinone (TIBO) belongs to non-nucleoside group of reverse transcriptase inhibitors (NNRTIs). A computational chemistry study has been performed on a series of tetrahydroimidazo-benzodiazepinones as HIV-1-NNRT inhibitors. Problem statement: In order to search out new drug of desired activity from the lead compounds, there was need to know the interaction of drugs with their receptor i.e., type of force(s) that have predominant role. Approach: Log P and SASA have been used for measurement of hydrophobic interaction, energy of protonation for measurement of most favorable hydrogen bond acceptor site, bond length and bond strain for measurement of strength of hydrogen bond formed between drug and receptor, atomic charges, ionization potential, electronegativity, $E^{\ddagger} n$ and $E^{\ddagger} m$ and their difference $\Delta \mathrm{E}^{\ddagger} \mathrm{nm}$ for measurement of polar interaction. The 3D modeling and geometry optimization of the compounds and receptor amino acids have been done by semiempirical method with MOPAC2002 associated with CAChe software. Results: The study has shown that hydrophobic interaction is predominant and made major contribution, while hydrogen bonding and polar interactions help in proper orientation of the compound (or its functional groups) to make maximam interaction. Conclusion: In this study theoretical technique has been discussed by which new hypothetical HIV-1NNRT inhibitors can be developed prior to their synthesis only by introducing effective hydrophobic substituents at specific sites.
\end{abstract}

Key words: Tetrahydroimidazobenzodiazepinones, NNRTIs binding pocket, hydrophobicity, hydrogen bond and effective atomic softness

\section{INTRODUCTION}

The binding of the drug (compound) to the receptor will initially depend upon the types of chemical bonds (covalent bond, ionic bond, hydrogen bond and hydrophobic interactions) that can be established between the drug and its receptor. The overall strengths of these bonds will vary and will determine the degree of affinity between the drug and the receptor. The affinity of the compound for the receptor is dependent upon its proper three-dimensional characteristics such as: its size, stereochemical orientation of its functional groups and its physical and electrochemical properties. In this study we have chosen twenty-one tetrahydroimidazobenzodiazepinone (TIBO) derivatives for drug-receptor interaction. TIBO belongs to nonnucleoside group of reversetranscriptase inhibitors (NNRTIs). The NNRTIs interect non-competitively with an allosteric site of the reverse transcriptase enzyme and thus do not directly impair the function of the substrate binding site ${ }^{[1]}$. In fact, NNRTIs have a comparatively higher binding affinity for the enzymesubstrate complex than for the free enzyme itself. Their interaction with the enzyme leads to a conformational change in the enzyme, resulting in a decrease in the affinity of the active site for the substrate. However, NNRTIs are active against the RT of only HIV-1 and not of HIV-2 or any other retrovirus. This specificity of NNRTIs for the HIV-1-RT is due to presence in HIV-1RT and not in other RTs or DNA polymerases, of a flexible highly hydrophobic pocket in which a nonsubstrate analogue can fit snugly ${ }^{[2-4]}$. The hydrophobic pocket in HIV-1-RT is formed by the hydrophobic residues (Y181, Y184, Y187 and Y188) of the Y181Y188 region ${ }^{[5]}$. The hydrophobic nature of the NNRTIs pocket provides relatively few possibilities for polar interaction and hydrogen bonding. In this article, we have studied various forces governing the drug-

Corresponding Author: V.K. Sahu, Department of Chemistry, Maharani Lal Kunwari (PG) College, Balrampur, Uttar Pradesh, 271201, India Tel: +919335236117 
receptor interaction of a series of TIBO derivatives ${ }^{[6]}$ with their receptor (NNRTIs binding pocket) ${ }^{[5,7]}$. The amino acids constituting the NNRTIs-pocket are Val (Y187), Met (Y184) and Tyr (Y181 and Y188). Since, Val and Met are hydrophobic in nature ${ }^{[8]}$, they must play a major role in hydrophobic interaction. To analyze hydrophobic interaction ${ }^{[9]}$, we have evaluated Log P and SASA of the substituents of each derivative and their effect on the activity of the compounds ${ }^{[10-12]}$. The hydrophobic nature of the NNRTIs pocket provides relatively few possibilities for polar interaction and hydrogen bonding. Amino acid, Tyr having phenolic group as its side chain only responsible for hydrogen bonding ${ }^{[13]}$. To analyze hydrogen bonding, we have searched out hydrogen bond donor and acceptor sites $^{[14]}$. Then, the strength of hydrogen bonds formed between the most favorable hydrogen acceptor and donor sites have been evaluated by bond length and bond strain. The hydrophobic nature of the NNRTIs pocket provides relatively few possibilities for polar interaction. To analyze polar interaction, we have evaluated atomic charges, ionization potential, electronegativity, acidic and basic atomic softness.

Theory: Te values of the above parameters have been evaluated by using the various equations given below. The Molecular Lipophilic Potential (MLP) ${ }^{[15]}$ was the first method designed to calculate the hydrophobic profile of a molecule in thre dimensions. The development of the MLP was based on the finding that the partition coefficient (P) of a molecule, which represents its relative distribution over an octanol/water boundary, can be estimated from its chemical structure $^{[10]}$ :

$$
\log P=\log \left[\frac{\text { Concentraton of during in oc tan ol }}{\text { Concentration of during in water }}\right]
$$

From the assumption that the $\log \mathrm{P}$ is an additive property of the molecular fragments that make up a molecule, values for a wide variety of atom types and groups have been calculated:

$$
\pi_{\mathrm{R}}=\log \mathrm{P}-\log \mathrm{P}_{\mathrm{H}}
$$

Where:

$\pi_{\mathrm{R}} \quad=$ The hydrophobicity of substituent- $\mathrm{R}$

$\log \mathrm{P}=$ The hydrophobicity of the whole compound

$\log \mathrm{P}_{\mathrm{H}}=$ The hydrophobicity of the compound when substituent-R is replaced by hydrogen atom:

$$
\pi_{\mathrm{X}},=\log \mathrm{P}_{\mathrm{R}}-\log \mathrm{P}_{\mathrm{RX}}
$$

Where:

$\pi_{\mathrm{X}}, \quad=$ The hydrophobicity of substituent-X'

$\log P_{R}=$ The hydrophobicity of the compound where substituent- $\mathrm{R}$ is replaced by hydrogen

$\log \mathrm{P}_{\mathrm{RX}}$, = The hydrophobicity of the compound when substituent-R and $X^{\prime}$, both are replaced by hydrogen atoms

One way to provide a simple account of surface properties is to compute the solvent accessible surface area (SASA) ${ }^{[11]}$. SASA was first described by Lee and Richards ${ }^{[11]}$ is sometimes called Lee-Richard molecular surface. SASA is typically calculated using the rolling ball algorithm developed by ${ }^{[12]}$. This approach provides a useful tool to gain insight into the over all extent of a hydrophobic region on a molecule or in the binding site of a protein but lacks any real account of the particular atom types that make up the binding site or their positions relative to one another. In addition, it provides no means of assessing the shape of the binding since, it only calculates the relative accessibility of the contributing atoms:

$$
{ }^{\mathrm{R}} \mathrm{SASA}=\mathrm{SASA}-{ }^{\mathrm{H}} \mathrm{SASA}
$$

Where:

${ }^{\mathrm{R}} \mathrm{SASA}=$ The solvent accessible surface area of substient-R, SASA is the solvent accessible surface area of the whole compound

${ }^{\mathrm{H}} \mathrm{SASA}=$ The solvent accessible surface area of the compound where substituent- $R$ is replaced by hydrogen atom:

${ }^{x^{\prime}}$ SASA $={ }^{\mathrm{R}}$ SASA $-{ }^{\mathrm{RX}}$ SASA

Where:

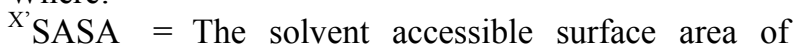
substient-X

${ }^{\mathrm{R}}$ SASA $=$ The solventaccessible surface area of the compound where substituent- $\mathrm{R}$ is replaced by hydrogen atom

${ }^{\mathrm{RX}}$ 'SASA $=$ The solventaccessible surface area of the compound where substituent-R and $\mathrm{X}$ ', both are replaced by hydrogen atoms

The total energy calculated by semiempirical methods has been shown to be a good descriptor in a number of different cases ${ }^{[16-19]}$. The total energy of a molecular system is the sum of the total electronic energy $\left(E_{\text {ee. }}\right)$ and the energy of internuclear repulsion $\left(\mathrm{E}_{\mathrm{nr}}\right)$ : 


$$
\text { Total Energy }(\mathrm{TE})=\mathrm{E}_{\mathrm{ee}}+\mathrm{E}_{\mathrm{nr}} .
$$

The energy of protonation defined as the difference between the total energies of the protonated and neutral forms of the molecule can be considered as a good measure of the strength of hydrogen bonds (the higher the energy, the stronger the bond) and can be used to determine the correct localization of the most favorable hydrogen bond acceptor site ${ }^{[14]}$ :

$$
\Delta \mathrm{TE}=\mathrm{TE}^{\prime}-\mathrm{TE}
$$

where, $\Delta \mathrm{TE}$ is the energy of protonation, TE is the total energy of neutral compound and TE' is the energy of protonated compound at a particular hydrogen acceptor site.

The softness of an atom in a molecule was described by ${ }^{[20]}$ and modified by ${ }^{[21]}$ The Klopman equation is given by:

$$
\begin{aligned}
& \mathrm{E}_{\mathrm{n}}^{*}=\mathrm{IP}_{\mathrm{n}}-\mathrm{b}^{2}\left(\mathrm{IP}_{\mathrm{n}}-\mathrm{EA}_{\mathrm{n}}\right)-\left[\chi_{\mathrm{s}}\left(\mathrm{C}_{\mathrm{s}}\right)^{2}\right. \\
& \left./ \mathrm{R}_{\mathrm{s}}\right](1-1 / \varepsilon) \times\left[\mathrm{q}_{\mathrm{s}}-2 \mathrm{~b}^{2} \chi_{\mathrm{s}}\left(\mathrm{C}_{\mathrm{s}}{ }^{\mathrm{n}}\right)^{2}\right] \\
& \mathrm{E}_{\mathrm{m}}^{*}=\mathrm{IP}_{\mathrm{m}}-\mathrm{-a}^{2}\left(\mathrm{IP}_{\mathrm{m}}-\mathrm{EA}_{\mathrm{m}}\right)-\left[\chi_{\mathrm{r}}\left(\mathrm{C}_{\mathrm{r}}^{\mathrm{m}}\right)^{2} /\right. \\
& \left.\mathrm{R}_{\mathrm{r}}\right](1-1 / \mathrm{e}) \times\left[\mathrm{q}_{\mathrm{r}}+2 \mathrm{~b}^{2} \chi_{\mathrm{r}}\left(\mathrm{C}_{\mathrm{r}}^{\mathrm{m}}\right)^{2}\right]
\end{aligned}
$$

Where:

$\mathrm{E}_{\mathrm{n}}^{\ddagger}=$ The softness of Lewis acid

$\mathrm{E}_{\mathrm{m}}{ }^{\ddagger}=$ The softness of a Lewis base

IP = The ionization potential of atom

$\mathrm{EA}=$ The electron affinity of atom

$\epsilon \quad=$ The dielectric constant of the medium in which reaction is carried out

$\mathrm{R}, \mathrm{q}=$ The radius and charge of atom $\mathrm{s}$ and $\mathrm{r}$

$\mathrm{C}=$ The electron density

$\chi \quad=\mathrm{q}-(\mathrm{q}-1) \sqrt{\mathrm{k}}$ and $\mathrm{k}=0.75$

$\mathrm{a}, \mathrm{b}=$ The variational parameter defined as $\mathrm{a}^{2}+\mathrm{b}^{2}=1$

It is well established that the stability of the compound formed between nucleophile and electrophile depends upon the value of difference between softness values of $E^{*} \mathrm{~m}$ of nucleophile and softness values of $E^{\star} n$ of electrophile, $\Delta E^{\star} n$ m represent the difference. The higher is the $\Delta \mathrm{E}^{\ddagger} \mathrm{nm}$ greater is the stability of the compound ${ }^{[22-24]}$ :

$$
\Delta E^{\star} \mathrm{nm}=\left|\mathrm{E}^{\star} \mathrm{n}-\mathrm{E}^{\star} \mathrm{m}\right|
$$

The method for the calculation of Ionization Potential of an atom in a molecule (IP) has been described by Dewar and Morita ${ }^{[25]}$ by the following equation:

$$
\text { IP }=a+b q+c q^{2}
$$

Where:

$\mathrm{q}=$ The charge of an atom in a molecule

$\mathrm{C}=$ The electron density of an atom in a molecule

The method for calculation of the Electron Affinity of an atom in a molecule (EA) is given as ${ }^{[26]}$ :

$$
\mathrm{EA}=-(\varepsilon \mathrm{HOMO}+\varepsilon \mathrm{LUMO})-(\mathrm{IP})
$$

where, HOMO and LUMO are the highest occupied and lowest unoccupied molecular orbital, respectively.

\section{MATERIALS AND METHODS}

Twentyone tetrahydroimidazobenzodiazepinone derivatives, that have been taken from literature, used as study material and are shown in Table 1 along with their observed biological activity in terms of $\mathrm{EC}_{50}$ (the concentration of compound leading to $50 \%$ effect and expressed in $\mathrm{mol} \mathrm{L}^{-1}$ or $\mathrm{mol} \mathrm{g}^{-1}$ ). The logarithms of the inverse of $\mathrm{EC}_{50}$ have been used as biological end point $\left(\log 1-\mathrm{C}^{-1}\right)$ in the study. For drug-receptor interaction, the molecular modeling and geometry optimization of all the derivatives have been carried out with CAChe Pro software by applying semiemperical method usimg MOPAC 2002. The parameters used for drug-receptor interaction: $\log \mathrm{P}$, solvent accessible surface area, energy of proto nation, bond length, bond order, bond strain, atomic charges and atomic softness have also been evaluated by solving the various equations given in the theory same software. $\log \mathrm{P}$ is calculated using the atom-typing scheme of Ghose and Crippen ${ }^{[27]}$. The solvent accessible surface area (SASA) is calculated at an optimized geometry in water. The water geometry is from optimization first using Augmented MM2, then using MOPAC with PM3 parameters and the conductor like screening model $(\mathrm{COSMO})^{[28]}$. The total energy is determined by a ZINDO calculation using INDO/1 parameters, at a geometry determined by optimization first with Augmented MM2 and then with MOPAC using PM3 parameters ${ }^{[29]}$. The partial charge calculated for an atom from quantum mechanics. Atom partial charges are determined by first optimizing the molecular geometry using Augmented MM2, followed by MOPAC with AM1 parameters. Values for bond property are ones that existed in the chemical sample when the extraction was evaluated. The fractional bond order (the distance between two bonded atoms) calculated from quantum mechanics. Bond orders are determined after geometry optimization using Augmented MM3 followed by MOPAC with PM3 parameters. The amount of steric (molecular mechanics) energy required to change the bond to its current length. 
Table 1: TIBO derivatives with their biological activity in terms of $\mathrm{EC}_{50}$
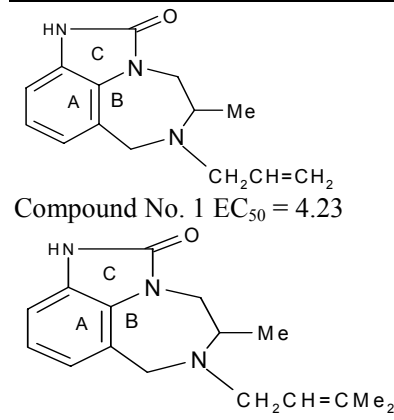

Compound No. $4 \mathrm{EC}_{50}=5.38$

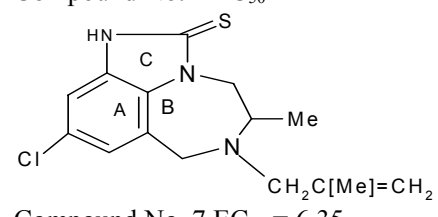

Compound No. $7 \mathrm{EC}_{50}=6.35$

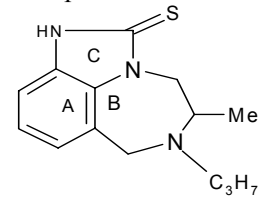

Compound No. $10 \mathrm{EC}_{50}=6.62$

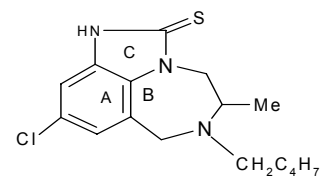

Compound No. $13 \mathrm{EC}_{50}=7.37$

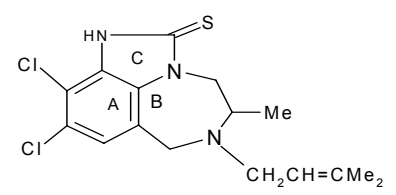

Compound No. $16 \mathrm{EC}_{50}=7.60$

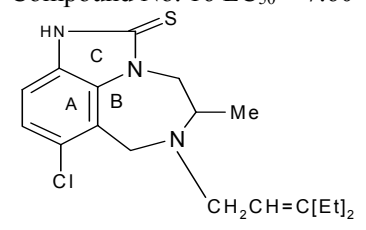

Compound No. $19 \mathrm{EC}_{50}=8.29$

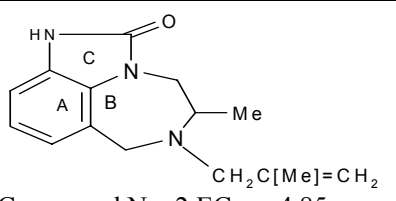

Compound No. $2 \mathrm{EC}_{50}=4.85$

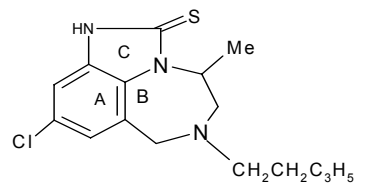

Compound No. $5 \mathrm{EC}_{50}=5.66$

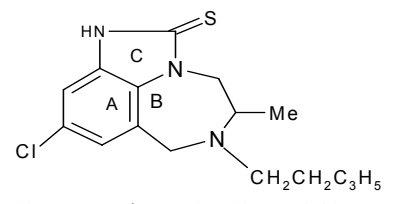

Compound No. $8 \mathrm{EC}_{50}=6.48$

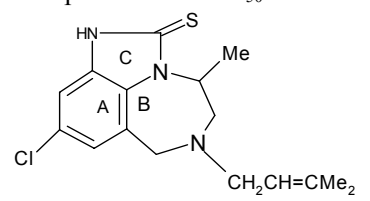

Compound No. $11 \mathrm{EC}_{50}=7.04$

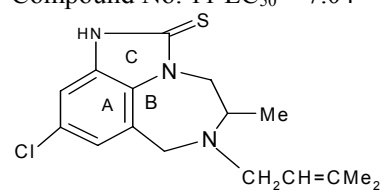

Compound No. $14 \mathrm{EC}_{50}=7.48$

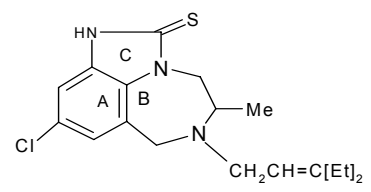

Compound No. $17 \mathrm{EC}_{50}=7.82$

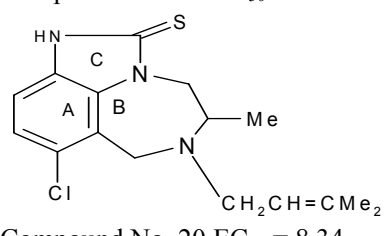

Compound No. $20 \mathrm{EC}_{50}=8.34$

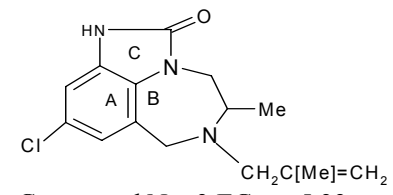

Compound No. $3 \mathrm{EC}_{50}=5.33$

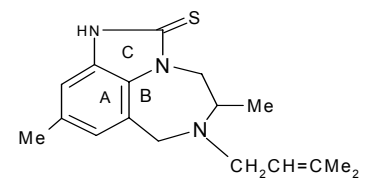

Compound No. $6 \mathrm{EC}_{50}=6.10$

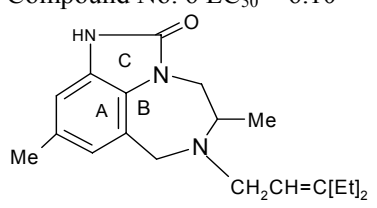

Compound No. $9 \mathrm{EC}_{50}=6.51$

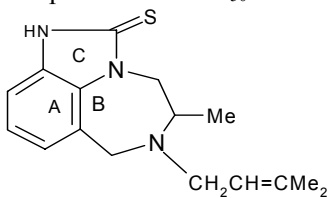

Compound No. $12 \mathrm{EC}_{50}=7.36$

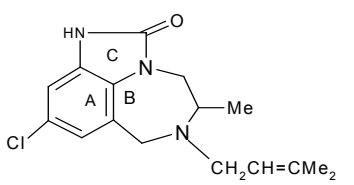

Compound No. $15 \mathrm{EC}_{50}=7.60$

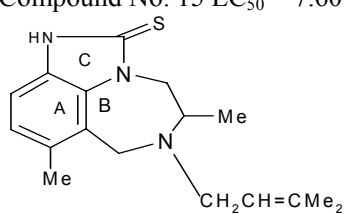

Compound No. $18 \mathrm{EC}_{50}=7.85$

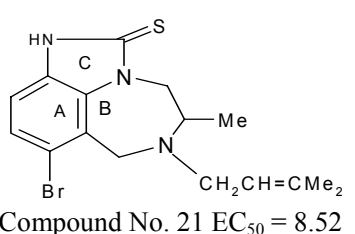

Bond strain energies are determined after optimization using Augmented MM2. The atomic softness of every atom of all the derivatives has been done by Softness Calculator (It is a program in basic language created by us used for the calculation of hardness, softness, electronegativity, chemical potential, $\mathrm{E}_{\mathrm{n}}{ }^{\dagger}$ and $\mathrm{E}_{\mathrm{m}}{ }^{\dagger}$ with the help of above equations) by semiemperical methods. The reaction medium has been consider fresh water hence dielectric constant $(\epsilon)$ has been taken for fresh water $81^{[30]}$.

\section{RESULTS}

The skeleton structure (Fig. 1) of TIBO is based on following parent skeleton, which have 10 sites. Tetrahydroimidazobenzodiazepinone derivatives are shwon in Table 1, alongwith their observed biological activities in terms of $\mathrm{EC}_{50}$ values, as reported by ${ }^{[1]}$. The values of $\log \mathrm{P}$ and SASA of the hydrophobic substituents of all the derivatives have been calculated and are shown in Table 2 and 3, respectively while, 
Am. J. Immunol., 4 (3): 33-42, 2008

Table 2: Calculation of $\log \mathrm{P}$ of the substituents of TIBO derivatives

\begin{tabular}{|c|c|c|c|c|c|c|c|}
\hline \multicolumn{4}{|c|}{ Log P at R-Substituent } & \multicolumn{4}{|c|}{ Log $P$ at $X^{\prime}$-Substituent } \\
\hline No. & $\log P$ & $\log P_{H}$ & $\pi_{\mathrm{R}}$ & $\mathrm{S}$. & $\log P_{R}$ & $\log P_{\mathrm{RX}}$ & $\pi_{\mathrm{X}}$ \\
\hline 1 & 1.265 & 0.163 & 1.102 & 1 & 0.163 & -0.250 & -0.087 \\
\hline 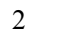 & 1.419 & 0.163 & 1.256 & 2 & 0.163 & -0.250 & -0.087 \\
\hline & 1.937 & 0.681 & 1.256 & & 0.681 & 0.268 & 0.413 \\
\hline 4 & 1.770 & 0.16 & 1.607 & & 0.163 & & -0.087 \\
\hline 5 & 3.432 & & & 5 & & & 0.413 \\
\hline & & & & & & & .414 \\
\hline & 0 & & 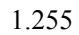 & & & & .413 \\
\hline & 4.326 & 2.361 & 1.965 & & 2.361 & 48 & 0.413 \\
\hline & 3.03 & 0.63 & 2.399 & & 0.6 & & 0.414 \\
\hline 10 & 3 & 1.8 & 1.172 & 10 & & & 0.413 \\
\hline 11 & & & 1.606 & 11 & & & 0.931 \\
\hline 12 & & & 66 & 12 & & & 413 \\
\hline 13 & 3 & 2.3 & 1. & 13 & 2.3 & 1. & 0.413 \\
\hline 14 & 3.967 & 2.361 & 1.606 & 14 & 2.361 & 1.948 & 0.413 \\
\hline 15 & 2.288 & 0.681 & 1.607 & 15 & 0.681 & 0.268 & 0.413 \\
\hline 16 & 4.485 & 2.87 & 1.606 & 16 & 2.879 & 2.466 & 0.413 \\
\hline 17 & 4.760 & 2.361 & 2.399 & 17 & 2.361 & 1.948 & 0.413 \\
\hline 18 & 3.917 & 2.310 & 1.607 & 18 & 2.310 & 1.897 & 0.413 \\
\hline 19 & & 2.36 & 2.399 & 19 & 2.361 & & 0.413 \\
\hline 20 & & & & 20 & & & 0.413 \\
\hline 21 & 4.241 & 2.635 & 1.606 & 21 & 2.635 & 2.221 & 0.413 \\
\hline
\end{tabular}

Table 3: Calculation of Solvent Accessible Surface Area (SASA) of the substituents of TIBO derivatives

\begin{tabular}{|c|c|c|c|c|c|c|c|}
\hline \multicolumn{4}{|c|}{ SASA at R-Substituent } & \multicolumn{4}{|c|}{ SASA at $X^{\prime}$-Substituent } \\
\hline No. & SASA & SASA & ${ }^{\mathrm{R}} \mathrm{SASA}$ & No. & . & SASA & ${ }^{x}$ SASA \\
\hline 1 & 113.731 & 95.558 & 18.173 & 1 & 5.558 & 90.617 & 4.941 \\
\hline & 118.740 & 95.698 & 42 & 2 & 98 & 90 & 981 \\
\hline & 130.086 & 107.004 & 82 & 3 & 107.004 & 101.933 & 5.071 \\
\hline & 126.198 & 95.710 & 30.4 & 4 & 5.710 & 90 & 089 \\
\hline 5 & 137.395 & 115.401 & & 5 & & 110 & 5.110 \\
\hline & 133. & 102 & & 0 & & & \\
\hline & & & & 7 & & & \\
\hline & 14 & 11 & & 8 & 0 & 11 & 4.943 \\
\hline & 145.969 & 103. & 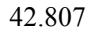 & , & ? & 98.298 & .864 \\
\hline 10 & 123.043 & 103. & 19.513 & 10 & 10 & 98 & 4.7 \\
\hline 11 & 147.275 & 116.506 & 30. & 11 & 116 & 98 & 17.670 \\
\hline 12 & 136. & 105 & & 12 & 10 & & 6.263 \\
\hline 13 & 143 & & & 13 & & & 1.986 \\
\hline 14 & & & & 4 & & & \\
\hline 15 & 139. & 108 & 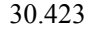 & 15 & 10 & 10 & 5.5 \\
\hline 16 & 152.695 & 122. & 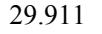 & 16 & 122 & 118 & 4.0 \\
\hline 17 & 159.151 & 116.261 & 42. & 17 & 116.261 & 110 & 62 \\
\hline 18 & 138.567 & 110.662 & 27.905 & 18 & 110.662 & 104.442 & 6.220 \\
\hline 19 & 158.331 & 114.653 & 43.678 & 19 & 114.653 & 108.614 & 6.039 \\
\hline 20 & 143.533 & 114.902 & 28.631 & 20 & 114.902 & 108.388 & 6.514 \\
\hline 21 & 148.592 & 118.475 & 30.117 & 21 & 118.475 & 112.085 & 6.390 \\
\hline
\end{tabular}

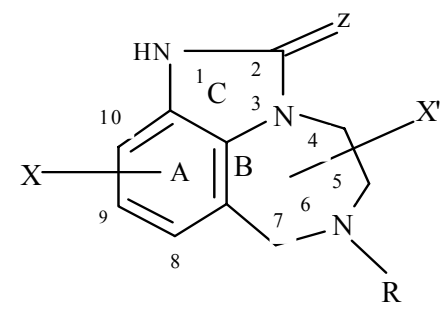

Fig. 1: Parent Skeleton of TIBO
Table 4: Calculation of hydrophobic parameters of TIBO drivatives and their relationships with observed activity $\left(\mathrm{EC}_{50}\right)$

Relationship between $\log$ P $\quad$ Relationship between SASA and and $\mathrm{EC}_{50} \quad \mathrm{EC}_{50}$

\begin{tabular}{|c|c|c|c|c|c|}
\hline & \\
\hline No. & $\log P$ & $\mathrm{EC}_{50}$ & S. No. & SASA & $\mathrm{EC}_{50}$ \\
\hline \multicolumn{6}{|c|}{ Subgroup-A } \\
\hline 21 & 4.241 & 8.52 & 17 & 159.151 & 7.82 \\
\hline 20 & 3.967 & 8.34 & 16 & 152.695 & 7.60 \\
\hline 14 & 3.967 & 7.48 & 14 & 148.072 & 7.48 \\
\hline 13 & 3.828 & 7.37 & 13 & 143.629 & 7.37 \\
\hline 12 & 3.449 & 7.36 & 12 & 136.434 & 7.36 \\
\hline 9 & 3.030 & 6.51 & 7 & 136.124 & 6.35 \\
\hline 6 & 2.237 & 6.10 & 6 & 133.326 & 6.10 \\
\hline 4 & 1.770 & 5.38 & 3 & 130.086 & 5.33 \\
\hline \multirow[t]{2}{*}{2} & 1.419 & 4.85 & 2 & 118.740 & 4.85 \\
\hline & & & 1 & 113.731 & 4.23 \\
\hline
\end{tabular}

Subgroup-B

\begin{tabular}{|c|c|c|c|c|c|}
\hline 19 & 4760 & 829 & & & \\
\hline 17 & 4.760 & 7.82 & 21 & 148.592 & 8.52 \\
\hline 16 & 4.485 & 7.60 & 20 & 143.533 & 8.34 \\
\hline 8 & 4.326 & 6.48 & 18 & 138.567 & 7.85 \\
\hline 7 & 3.616 & 6.35 & 5 & 137.395 & 5.66 \\
\hline 10 & 3.015 & 6.62 & 4 & 126.198 & 5.38 \\
\hline Subs & $\mathrm{p}-\mathrm{C}$ & & & & \\
\hline 18 & 3.917 & 7.85 & 19 & 158.331 & 8.29 \\
\hline 5 & 3.432 & 5.66 & 11 & 147.275 & 7.04 \\
\hline 15 & 2.288 & 7.60 & 9 & 145.969 & 6.51 \\
\hline 3 & 1.937 & 5.33 & 10 & 123.043 & 6.62 \\
\hline 1 & 1.265 & 4.23 & $8^{\mathrm{P}}$ & 149.633 & 6.48 \\
\hline $11^{\mathrm{P}}$ & 3.967 & 7.04 & $15^{\mathrm{P}}$ & 139.046 & 7.60 \\
\hline
\end{tabular}

P: Indicates compounds do not follow sequential trend

Table 5: Calculation of energy of protonation of hydrogen bond acceptors on TIBO derivatives

Energy of protonation at site-3 Energy of Protonation at site-6

\begin{tabular}{llllllll}
\multicolumn{2}{l}{ No. TE } & TE & $\Delta$ TE & \multicolumn{2}{l}{ No. TE } & TE' & $\Delta$ TE \\
\hline 1 & -129.31 & -129.35 & 0.036 & 1 & -129.35 & -128.64 & 0.708 \\
2 & -136.50 & -136.54 & 0.036 & 2 & -136.54 & -135.83 & 0.710 \\
3 & -148.27 & -148.30 & 0.032 & 3 & -148.30 & -147.60 & 0.708 \\
4 & -143.68 & -143.72 & 0.037 & 4 & -143.72 & -143.01 & 0.710 \\
5 & -145.69 & -145.73 & 0.043 & 5 & -145.73 & -145.03 & 0.699 \\
6 & -150.88 & -150.91 & 0.033 & 6 & -150.91 & -150.20 & 0.712 \\
7 & -145.43 & -145.46 & 0.028 & 7 & -145.46 & -144.78 & 0.680 \\
8 & -154.40 & -154.45 & 0.046 & 8 & -154.45 & -153.76 & 0.689 \\
9 & -165.19 & -165.23 & 0.038 & 9 & -165.23 & -164.51 & 0.713 \\
10 & -128.31 & -128.36 & 0.049 & 10 & -128.36 & -127.67 & 0.692 \\
11 & -152.79 & -153.01 & 0.218 & 11 & -153.01 & -151.97 & 1.039 \\
12 & -140.84 & -140.90 & 0.060 & 12 & -140.90 & -140.20 & 0.695 \\
13 & -152.92 & -152.96 & 0.047 & 13 & -152.96 & -152.27 & 0.693 \\
14 & -152.61 & -152.66 & 0.048 & 14 & -152.66 & -151.97 & 0.688 \\
15 & -155.46 & -155.49 & 0.037 & 15 & -155.49 & -154.78 & 0.709 \\
16 & -164.37 & -164.43 & 0.061 & 16 & -164.43 & -163.73 & 0.701 \\
17 & -166.92 & -166.98 & 0.057 & 17 & -166.98 & -166.28 & 0.695 \\
18 & -148.02 & -148.08 & 0.053 & 18 & -148.08 & -147.38 & 0.697 \\
19 & -166.90 & -166.97 & 0.071 & 19 & -166.97 & -166.28 & 0.689 \\
20 & -152.60 & -152.66 & 0.061 & 20 & -152.66 & -151.96 & 0.701 \\
21 & -150.72 & -150.77 & 0.055 & 21 & -150.77 & -150.09 & 0.681 \\
\hline
\end{tabular}

Table 4 shows their relationships with observed activity $\left(\mathrm{EC}_{50}\right)$. To analyze hydrogen bond interaction, we have evaluated energy of protonation to identify the hydrogen bond acceptors and to measure the most favourable hydrogen bondings and are shown in Table 5. 
Am. J. Immunol., 4 (3): 33-42, 2008

Table 6: Calculation of bond properties of Hydrogen Bond (H-Bond) formed between nitrogen at Site- 6 and hydrogen of side chain of Tyrosine (Y188)

\begin{tabular}{llllll}
\hline No. & H-bond & $\mathrm{N}^{\delta-}$ & $\mathrm{H}^{\delta+}$ & $\begin{array}{l}\text { Bond } \\
\text { length }\end{array}$ & $\begin{array}{c}\text { Bond } \\
\text { strain }\end{array}$ \\
\hline 1 & ${ }^{\delta-} \mathrm{N}----\mathrm{H}^{\delta+}$ & -0.292 & 0.240 & 2.514 & 0.007 \\
2 & ${ }^{\delta-} \mathrm{N}----\mathrm{H}^{\delta+}$ & -0.318 & 0.234 & 2.532 & 0.234 \\
3 & ${ }^{\delta-} \mathrm{N}----\mathrm{H}^{\delta+}$ & -0.326 & 0.235 & 2.537 & 0.011 \\
4 & ${ }^{\delta-} \mathrm{N}----\mathrm{H}^{\delta+}$ & -0.275 & 0.240 & 2.700 & 0.021 \\
5 & ${ }^{\delta-} \mathrm{N}----\mathrm{H}^{\delta+}$ & -0.284 & 0.237 & 4.097 & 0.035 \\
6 & ${ }^{\delta-} \mathrm{N}----\mathrm{H}^{\delta+}$ & -0.274 & 0.230 & 4.893 & 0.049 \\
7 & ${ }^{\delta-} \mathrm{N}----\mathrm{H}^{\delta+}$ & -0.259 & 0.233 & 2.936 & 0.075 \\
8 & ${ }^{\delta-} \mathrm{N}----\mathrm{H}^{\delta+}$ & -0.288 & 0.235 & 3.553 & 0.154 \\
9 & ${ }^{\delta-} \mathrm{N}----\mathrm{H}^{\delta+}$ & -0.295 & 0.241 & 2.539 & 0.019 \\
10 & ${ }^{\delta-} \mathrm{N}----\mathrm{H}^{\delta+}$ & -0.284 & 0.227 & 4.262 & 0.235 \\
11 & ${ }^{\delta-} \mathrm{N}----\mathrm{H}^{\delta+}$ & -0.280 & 0.238 & 2.653 & 0.018 \\
12 & ${ }^{\delta-} \mathrm{N}----\mathrm{H}^{\delta+}$ & -0.275 & 0.236 & 2.512 & 0.105 \\
13 & ${ }^{\delta-} \mathrm{N}----\mathrm{H}^{\delta+}$ & -0.277 & 0.236 & 4.218 & 0.051 \\
14 & ${ }^{\delta-} \mathrm{N}----\mathrm{H}^{\delta+}$ & -0.274 & 0.239 & 2.628 & 0.031 \\
15 & ${ }^{\delta-} \mathrm{N}----\mathrm{H}^{\delta+}$ & -0.278 & 0.235 & 3.676 & 0.123 \\
16 & ${ }^{\delta-} \mathrm{N}----\mathrm{H}^{\delta+}$ & -0.295 & 0.235 & 4.551 & 0.001 \\
17 & ${ }^{\delta-} \mathrm{N}----\mathrm{H}^{\delta+}$ & -0.281 & 0.239 & 4.063 & 0.050 \\
18 & ${ }^{\delta-} \mathrm{N}----\mathrm{H}^{\delta+}$ & -0.282 & 0.239 & 3.976 & 0.011 \\
19 & ${ }^{\delta-} \mathrm{N}----\mathrm{H}^{\delta+}$ & -0.277 & 0.238 & 2.839 & 0.099 \\
20 & ${ }^{\delta-} \mathrm{N}----\mathrm{H}^{\delta+}$ & -0.277 & 0.235 & 3.653 & 0.062 \\
21 & ${ }^{\delta-} \mathrm{N}----\mathrm{H}^{\delta+}$ & -0.277 & 0.236 & 3.970 & 0.091 \\
\hline
\end{tabular}

The bond properties of various hydrogen bonds (formed between nitrogen at site- 6 and hydrogen of side chain of tyrosine residue at Y188, between nitrogen at site-3 and hydrogen of side chain of tyrosine residue at Y188 and between hydrogen at site-1 and oxygen of side chain of Tyrosine residue at Y181) have been evaluated and are shown in Table 6-8.
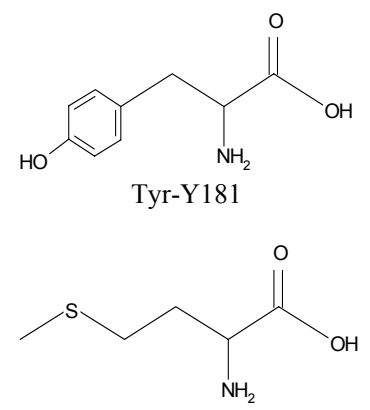

Met-Y184
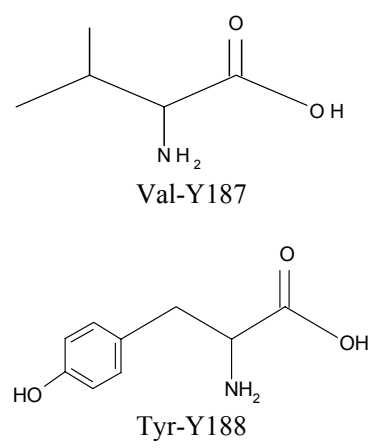

Table 7: Calculation of bond properties of Hydrogen Bond (H-Bond) formed between nitrogen at site- 3 and Hydrogen of side chain of Tyrosine (Y188) at site-3

\begin{tabular}{|c|c|c|c|c|c|}
\hline No. & H-bond & $\mathrm{N}^{\delta-}$ & $\mathrm{H}^{\mathrm{d}+}$ & $\begin{array}{l}\text { Bond } \\
\text { length }\end{array}$ & $\begin{array}{l}\text { Bond } \\
\text { strain }\end{array}$ \\
\hline 1 & ${ }^{\delta-} \mathrm{N}----\mathrm{H}^{\mathrm{d}+}$ & -0.223 & 0.249 & 3.742 & 9.0260 \\
\hline 2 & ${ }^{\delta-} \mathrm{N}----\mathrm{H}^{\mathrm{d}+}$ & -0.261 & 0.248 & 5.109 & 8.4420 \\
\hline 3 & ${ }^{\delta-} \mathrm{N}----\mathrm{H}^{\mathrm{d}+}$ & -0.227 & 0.245 & 4.534 & 7.8070 \\
\hline 4 & ${ }^{\delta-} \mathrm{N}----\mathrm{H}^{\mathrm{d}+}$ & -0.230 & 0.229 & 4.911 & 9.7060 \\
\hline 5 & ${ }^{\delta-} \mathrm{N}----\mathrm{H}^{\mathrm{d}+}$ & -0.178 & 0.219 & 4.258 & 9.8770 \\
\hline 6 & ${ }^{\delta-} \mathrm{N}----\mathrm{H}^{\mathrm{d}+}$ & -0.184 & 0.254 & 5.779 & 10.264 \\
\hline 7 & ${ }^{\delta-} \mathrm{N}----\mathrm{H}^{\mathrm{d}+}$ & -0.192 & 0.234 & 4.372 & 9.8130 \\
\hline 8 & ${ }^{\delta-} \mathrm{N}----\mathrm{H}^{\mathrm{d}+}$ & -0.178 & 0.246 & 4.453 & 10.139 \\
\hline 9 & ${ }^{\delta-} \mathrm{N}----\mathrm{H}^{\mathrm{d}+}$ & -0.216 & 0.246 & 3.745 & 8.7330 \\
\hline 10 & ${ }^{\delta-} \mathrm{N}----\mathrm{H}^{\mathrm{d}+}$ & -0.195 & 0.236 & 3.588 & 10.098 \\
\hline 11 & ${ }^{\delta-} \mathrm{N}----\mathrm{H}^{\mathrm{d}+}$ & -0.190 & 0.246 & 4.457 & 9.6490 \\
\hline 12 & ${ }^{\delta-} \mathrm{N}----\mathrm{H}^{\mathrm{d}+}$ & -0.188 & 0.249 & 4.054 & 9.7700 \\
\hline 13 & ${ }^{\delta-} \mathrm{N}----\mathrm{H}^{\mathrm{d}+}$ & -0.192 & 0.234 & 6.434 & 9.6900 \\
\hline 14 & ${ }^{\delta-} \mathrm{N}----\mathrm{H}^{\mathrm{d}+}$ & -0.189 & 0.246 & 4.457 & 9.6400 \\
\hline 15 & ${ }^{\delta-} \mathrm{N}----\mathrm{H}^{\mathrm{d}+}$ & -0.225 & 0.225 & 4.528 & 10.734 \\
\hline 16 & ${ }^{\delta-} \mathrm{N}----\mathrm{H}^{\mathrm{d}+}$ & -0.180 & 0.247 & 3.721 & 9.7240 \\
\hline 17 & ${ }^{\delta-} \mathrm{N}----\mathrm{H}^{\mathrm{d}+}$ & -0.197 & 0.233 & 3.675 & 9.8260 \\
\hline 18 & ${ }^{\delta-} \mathrm{N}----\mathrm{H}^{\mathrm{d}+}$ & -0.187 & 0.231 & 4.631 & 9.0360 \\
\hline 19 & ${ }^{\delta-} \mathrm{N}----\mathrm{H}^{\mathrm{d}+}$ & -0.186 & 0.243 & 4.485 & 9.7760 \\
\hline 20 & ${ }^{\delta-} \mathrm{N}----\mathrm{H}^{\mathrm{d}+}$ & -0.191 & 0.248 & 4.524 & 10.508 \\
\hline 21 & ${ }^{\delta-} \mathrm{N}----\mathrm{H}^{\mathrm{d}+}$ & -0.196 & 0.235 & 3.789 & 10.122 \\
\hline
\end{tabular}

Table 8: Calculation of bond properties of Hydrogen Bond (H-Bond) formed between hydrogen at site-1 and oxygen of side chain of Tyrosine (Y181)

\begin{tabular}{|c|c|c|c|c|c|}
\hline No. & H-Bond & $\mathrm{H}^{\delta+}$ & $\mathrm{O}^{\delta-}$ & $\begin{array}{l}\text { Bond } \\
\text { length }\end{array}$ & $\begin{array}{l}\text { Bond } \\
\text { strain }\end{array}$ \\
\hline 1 & ${ }^{\delta+} \mathrm{H}----\mathrm{O}^{\delta-}$ & 0.268 & -0.266 & 3.634 & 0.021 \\
\hline 2 & ${ }^{\delta+} \mathrm{H}----\mathrm{O}^{\delta-}$ & 0.282 & -0.245 & 2.782 & 0.064 \\
\hline 3 & ${ }^{\delta+} \mathrm{H}----\mathrm{O}^{\delta-}$ & 0.275 & -0.275 & 2.604 & 0.000 \\
\hline 4 & ${ }^{\delta+} \mathrm{H}----\mathrm{O}^{\delta-}$ & 0.273 & -0.267 & 2.945 & 0.024 \\
\hline 5 & ${ }^{\delta+} \mathrm{H}----\mathrm{O}^{\delta-}$ & 0.298 & -0.300 & 4.493 & 0.059 \\
\hline 6 & ${ }^{\delta+} \mathrm{H}----\mathrm{O}^{\delta-}$ & 0.297 & -0.300 & 1.852 & 0.052 \\
\hline 7 & ${ }^{\delta+} \mathrm{H}----\mathrm{O}^{\delta-}$ & 0.295 & -0.270 & 3.323 & 0.026 \\
\hline 8 & ${ }^{\delta+} \mathrm{H}----\mathrm{O}^{\delta-}$ & 0.301 & -0.283 & 3.259 & 0.021 \\
\hline 9 & ${ }^{\delta+} \mathrm{H}----\mathrm{O}^{\delta-}$ & 0.275 & -0.284 & 5.832 & 0.024 \\
\hline 10 & ${ }^{\delta+} \mathrm{H}----\mathrm{O}^{\delta-}$ & 0.296 & -0.300 & 2.496 & 0.006 \\
\hline 11 & ${ }^{\delta+} \mathrm{H}----\mathrm{O}^{\delta-}$ & 0.301 & -0.283 & 1.834 & 0.085 \\
\hline 12 & ${ }^{\delta+} \mathrm{H}----\mathrm{O}^{\delta-}$ & 0.288 & -0.268 & 3.433 & 0.025 \\
\hline 13 & ${ }^{\delta+} \mathrm{H}----\mathrm{O}^{\delta-}$ & 0.300 & -0.302 & 3.224 & 0.038 \\
\hline 14 & ${ }^{\delta+} \mathrm{H}----\mathrm{O}^{\delta-}$ & 0.298 & -0.301 & 2.662 & 0.026 \\
\hline 15 & ${ }^{\delta+} \mathrm{H}----\mathrm{O}^{\delta-}$ & 0.259 & -0.249 & 4.289 & 0.043 \\
\hline 16 & ${ }^{\delta+} \mathrm{H}----\mathrm{O}^{\delta-}$ & 0.306 & -0.279 & 1.819 & 0.026 \\
\hline 17 & ${ }^{\delta+} \mathrm{H}----\mathrm{O}^{\delta-}$ & 0.301 & -0.283 & 1.835 & 0.011 \\
\hline 18 & ${ }^{\delta+} \mathrm{H}----\mathrm{O}^{\delta-}$ & 0.298 & -0.281 & 1.837 & 0.020 \\
\hline 19 & ${ }^{\delta+} \mathrm{H}----\mathrm{O}^{\delta-}$ & 0.300 & -0.282 & 3.153 & 0.030 \\
\hline 20 & ${ }^{\delta+} \mathrm{H}----\mathrm{O}^{\delta-}$ & 0.299 & -0.301 & 1.848 & 0.062 \\
\hline 21 & ${ }^{\delta+} \mathrm{H}----\mathrm{O}^{\delta-}$ & 0.301 & -0.283 & 1.835 & 0.004 \\
\hline
\end{tabular}

For polar interaction, acidic atomic softness $\left(E^{*} n\right)$ and basic atomic softness $\left(\mathrm{E}^{\ddagger} \mathrm{m}\right)$ of the reactive sites of each derivative and their difference $\left(\Delta E^{\ddagger} \mathrm{nm}\right)$ has been evaluated and are shown in Table 9 and 10. 
Table 9: $\Delta \mathrm{E}_{\mathrm{nm}}^{\ddagger}$ values derived from $\mathrm{E}_{\mathrm{n}}^{\ddagger}$ of Carbon atom (-CONH-) of amino acids and $\mathrm{E}_{\mathrm{m}}^{\ddagger}$ of Oxygen (-CO-) and Sulphur (-CS-) atom of the compounds

\begin{tabular}{|c|c|c|c|c|c|}
\hline No. & A & $\mathrm{E}_{\mathrm{m}}^{\hbar}$ & $\begin{array}{l}\text { Tyr } \\
\mathrm{E}_{\mathrm{n}}^{\ddagger}=36.03969 \\
\Delta \mathrm{E}_{\mathrm{nm}}^{\ddagger}\end{array}$ & $\begin{array}{l}\text { Glu } \\
\mathrm{E}_{\mathrm{n}}^{\sharp}=36.00171 \\
\Delta \mathrm{E}_{\mathrm{nm}}^{\ddagger}\end{array}$ & $\begin{array}{l}\text { Asn } \\
E_{n}^{\ddagger}=36.08613 \\
\Delta E_{n m}^{\ddagger}\end{array}$ \\
\hline 1 & $\mathrm{O}$ & -21.5674 & 57.607 & 57.569 & 57.654 \\
\hline 2 & $\mathrm{O}$ & -21.5165 & 57.556 & 57.518 & 57.603 \\
\hline 3 & $\mathrm{O}$ & -21.2510 & 57.291 & 57.253 & 57.337 \\
\hline 4 & $\mathrm{O}$ & -21.6042 & 57.644 & 57.606 & 57.690 \\
\hline 5 & $\mathrm{~S}$ & -8.87875 & 44.918 & 44.880 & 44.965 \\
\hline 6 & $\mathrm{~S}$ & -9.02001 & 45.060 & 45.022 & 45.106 \\
\hline 7 & $\mathrm{~S}$ & -9.09032 & 45.130 & 45.092 & 45.176 \\
\hline 8 & $\mathrm{~S}$ & -8.64563 & 44.685 & 44.647 & 44.732 \\
\hline 9 & $\mathrm{O}$ & -21.7132 & 57.753 & 57.715 & 57.799 \\
\hline 10 & $\mathrm{~S}$ & -8.93743 & 44.977 & 44.939 & 45.024 \\
\hline 11 & $\mathrm{~S}$ & -8.64372 & 44.683 & 44.645 & 44.730 \\
\hline 12 & $\mathrm{~S}$ & -8.87625 & 44.916 & 44.878 & 44.962 \\
\hline 13 & $\mathrm{~S}$ & -8.69880 & 44.738 & 44.701 & 44.785 \\
\hline 14 & $\mathrm{~S}$ & -8.64575 & 44.685 & 44.647 & 44.732 \\
\hline 15 & $\mathrm{O}$ & -21.3191 & 57.359 & 57.321 & 57.405 \\
\hline 16 & $\mathrm{~S}$ & -8.43330 & 44.473 & 44.435 & 44.519 \\
\hline 17 & $\mathrm{~S}$ & -8.66098 & 44.701 & 44.663 & 44.747 \\
\hline 18 & $\mathrm{~S}$ & -9.40715 & 45.447 & 45.409 & 45.493 \\
\hline 19 & $\mathrm{~S}$ & -8.69364 & 44.733 & 44.695 & 44.780 \\
\hline 20 & $\mathrm{~S}$ & -9.15172 & 45.191 & 45.153 & 45.238 \\
\hline 21 & $\mathrm{~S}$ & -9.07213 & 45.112 & 45.074 & 45.158 \\
\hline
\end{tabular}

Table 10: $\Delta \mathrm{E}^{\ddagger}{ }_{\mathrm{nm}}$ values derived from $\mathrm{E}^{\ddagger}{ }_{\mathrm{n}}$ of Carbon (-CO-) and Sulphur (-CS-) of the compounds and $\mathrm{E}_{\mathrm{m}}^{\ddagger}$ of Oxygen atom of amino acids (-CONH-)

\begin{tabular}{|c|c|c|c|c|c|}
\hline No. & A & $\mathrm{E}_{\mathrm{n}}^{+}$ & $\begin{array}{l}\text { Tyr } \\
\mathrm{E}_{\mathrm{m}}^{\ddagger}=-22.3384 \\
\Delta \mathrm{E}_{\mathrm{nm}}^{\ddagger}\end{array}$ & $\begin{array}{l}\text { Glu } \\
\mathrm{E}_{\mathrm{m}}^{\ddagger}=-22.2171 \\
\Delta \mathrm{E}_{\mathrm{nm}}^{\ddagger}\end{array}$ & $\begin{array}{l}\text { Asn } \\
\mathrm{E}_{\mathrm{m}}^{\ddagger}=-22.5734 \\
\Delta \mathrm{E}_{\mathrm{nm}}^{\ddagger}\end{array}$ \\
\hline 1 & $\mathrm{C}$ & 41.9909 & 64.329 & 64.208 & 64.564 \\
\hline 2 & $\mathrm{C}$ & 41.9919 & 64.330 & 64.209 & 64.565 \\
\hline 3 & $\mathrm{C}$ & 42.0364 & 64.375 & 64.254 & 64.610 \\
\hline 4 & $\mathrm{C}$ & 41.9928 & 64.331 & 64.210 & 64.566 \\
\hline 5 & $\mathrm{C}$ & 56.8538 & 79.192 & 79.071 & 79.427 \\
\hline 6 & $\mathrm{C}$ & 56.8572 & 79.196 & 79.074 & 79.431 \\
\hline 7 & $\mathrm{C}$ & 57.1111 & 79.449 & 79.328 & 79.684 \\
\hline 8 & $\mathrm{C}$ & 56.8374 & 79.176 & 79.054 & 79.411 \\
\hline 9 & $\mathrm{C}$ & 41.9571 & 64.296 & 64.174 & 64.531 \\
\hline 10 & $\mathrm{C}$ & 56.8609 & 79.199 & 79.078 & 79.434 \\
\hline 11 & $\mathrm{C}$ & 56.8359 & 79.174 & 79.053 & 79.409 \\
\hline 12 & $\mathrm{C}$ & 56.7725 & 79.111 & 78.990 & 79.346 \\
\hline 13 & $\mathrm{C}$ & 56.9260 & 79.264 & 79.143 & 79.499 \\
\hline 14 & $\mathrm{C}$ & 56.8355 & 79.174 & 79.053 & 79.409 \\
\hline 15 & $\mathrm{C}$ & 41.9972 & 64.336 & 64.214 & 64.571 \\
\hline 16 & $\mathrm{C}$ & 56.5651 & 78.904 & 78.782 & 79.138 \\
\hline 17 & $\mathrm{C}$ & 56.8469 & 79.185 & 79.064 & 79.420 \\
\hline 18 & $\mathrm{C}$ & 56.9836 & 79.322 & 79.201 & 79.557 \\
\hline 19 & $\mathrm{C}$ & 56.9142 & 79.253 & 79.131 & 79.488 \\
\hline 20 & $\mathrm{C}$ & 57.1369 & 79.475 & 79.354 & 79.710 \\
\hline 21 & $\mathrm{C}$ & 41.9909 & 64.329 & 64.208 & 64.564 \\
\hline
\end{tabular}

\section{DISCUSSION}

Hydrophobic interactions play a crucial role in ligand-protein binding ${ }^{[31]}$. Most ligand binding sites contain at least one hydrophobic (nonpolar) region, with many demonstrating a clear preference for nonpolar ligands. In this case, out of four receptor's amino acids the two are hydrophobic (Met-Y184 and Val-Y187) in nature. In these, the valine (Val-Y187) amino acid is the second top most hydrophobic amino acid (first one is isoleucine) and is responsible for hydrophobic interaction with R-substituents of the compounds. While methionine (Met-Y184) is also hydrophobic in nature and its side chain has $\mathrm{CH}_{3}-\mathrm{S}$ fragment at the end, which is responsible hydrophobic interaction but with $\mathrm{X}^{\prime}$-substituents $\left(\mathrm{CH}_{3}\right.$-group) of the compounds. The substituent's hydrophobicity of all the derivatives have been calculated and are shown Table 2. Table 2 shows that $\mathrm{CH}_{2} \mathrm{CH}=\mathrm{C}[\mathrm{Et}]_{2}$ as $\mathrm{R}-$ substation's have highest value of $\log \mathrm{P}$ (compound no9, 17 and 19 having $\log \mathrm{P}=2.399), \mathrm{CH}_{2} \mathrm{CH}=\mathrm{C}[\mathrm{Me}]_{2}$ have a median values (compound no-6, 11, 12, 14, 16, 20 and 21 with $\log \mathrm{P}=1.606)$ and $\mathrm{CH}_{2} \mathrm{CH}_{2} \mathrm{C}_{3} \mathrm{H}_{5}$ have lowest value (compound no-5, $\log \mathrm{P}=1.071$ ) vale of $\log \mathrm{P}$. While $\mathrm{CH}_{3}$-group as $\mathrm{X}^{\prime}$-substituents has $\log \mathrm{P}$ value 0.413 . When both hydrophobic substituents-R and $\mathrm{X}^{\prime}$ have been removed, there is great loss in the hydrophobicity of the compounds as clear from the negative values of $\log \mathrm{P}$ of compounds-1, 2 and 4 . The negative value of $\log \mathrm{P}$ is an indication of hydrophilicity and loss of hydrophobicity. Thus, there must be a relationship between the hydrophobicity (log P) and activity of the drugs. A close look of Table 4 shows there is a direct relationship between the hydrophobicity $(\log$ P) and activity of the compounds and as $\log \mathrm{P}$ decreases activity decreases.

SASA also provides a useful tool to gain insight into the over all extent of a hydrophobic region on a molecule or in the binding site of a protein but lacks any real account of the particular atom types that make up the binding site or their positions relative to one another. In addition, it provides no means of assessing the shape of the binding, since it only calculates the relative accessibility of the contributing atoms. The substituent's SASA of all the derivatives have been calculated and are shown in Table 3 . Table 3 shows that $\mathrm{CH}_{2} \mathrm{CH}=\mathrm{C}[\mathrm{Et}]_{2}$ as $\mathrm{R}$-substituents have highest value of SASA (34.593-43.678), $\mathrm{CH}_{2} \mathrm{CH}=\mathrm{C}[\mathrm{Me}]_{2}$ have values (30.117-31.497) somewhat lower than values of $\mathrm{CH}_{2} \mathrm{CH}=\mathrm{C}[\mathrm{Et}]_{2}$. While $\mathrm{CH}_{2} \mathrm{CH} 2=\mathrm{CH}_{2}$ have lowest value (18.173) of SASA. $\mathrm{CH}_{3}$-group as $\mathrm{X}^{\prime}$-substituents has SASA value lower than R-substituents. A close look of Table 4 also shows that there is a direct relationship between the SASA and activity of the compounds and as SASA decreases activity decreases. For a large hydrophobic object, it becomes impossible to maintain a hydrogen-binding network in its vicinity resulting in the disruption of the structure of water and a stronger hydrophobic interaction. The Lum-Chandler Weeks theory of hydrophobicity can account for the 
transition that occurs from the hydrophobic hydration of small nonpolar solutes to the strong tendency for depletion of water near extended nonpolar surfaces of nanometer length scale such as those in proteins ${ }^{[32,33]}$. Consequently, the computer simultation evidence and recent theoretical developments reveal the need to capture the stronger hydrophobic attraction that would arise between a ligand and a protein with a large or concave nonpolar surface. The strength of the hydrophobic interaction is thus influenced not only by the polarity but also by the shape and extent of the exposed molecular surface.

Hydrogen bonding is most likely an essential requirement for many drug-receptor interactions. A single hydrogen bond is relatively weak and would not be expected to support a drug-receptor interaction alone, but when multiple hydrogen bonds are formed between drugs and receptors, as is typically the case, a significant amount of stability is conferred upon the drug-receptor interaction. The energy of protonation defined as the difference between the total energies of the protonated and neutral forms of the molecule can be considered as a good measure of the strength of hydrogen bonds (the higher the energy, the stronger the bond) and can be used to determine the correct localization of the most favorable hydrogen bond acceptor site $^{[14]}$. The TIBO derivatives have three nitrogen atoms, out of which two (at site-3 and 6) may act as hydrogen bond acceptor and the remaining one (at site-1) as donor. For correct localization of the most favorable hydrogen bond acceptor site, we have calculated energy of protonation of site- 3 and 6 and are shown in Table 5. Table shows that site- 6 is the most favorable hydrogen bond acceptor site as it has higher energy of protonation (ranging from 0.680-1.039) than site-3 (ranging from 0.028-0.218). In the hydrophobic pocket of the HIV-1-RTase, tyrosine amino acid constitutes the residues 181 and Y188. The phenolic $(-\mathrm{OH})$ group of the side chain of this amino acid has been evaluated to acts as hydrogen donor and thus formed $\mathrm{H}$-bond with $\mathrm{N}$-atom of site- 6 and or with site3 . The bond properties of the H-bonds formed have been evaluated and are shown into Table 6 and 7 . Table 6 and 7 shows the H-bond formed between Natom at site- 6 and $\mathrm{H}$-atom of Y188 residue have comparatively short bond length and lesser bond strain (most favourable $\mathrm{H}$-bond) than $\mathrm{H}$-bond formed between $\mathrm{N}$-atom at site-3. Another most favourable $\mathrm{H}$-bond is formed between $\mathrm{H}$-atom of hydrogen donor (-NH-) at site-1 and O-atom of the phenolic (-OH) group of the side chain of tyrosine amino acid at Y181. The bond properties of the bond as evaluated are shown in into Table 8.
The hydrophobic pocket in HIV-1-RT is formed by the hydrophobic residues (Y181, Y184, Y187 and Y188) of the Y181-Y188 regions. The hydrophobic nature of the NNRTIs pocket provides relatively few possibilities for polar interaction and hydrogen bonding. The remaining residues of the Y181-Y188 regions are Asn-Y182, Tyr-Y183, Glu-Y185 and GluY186 and constitute the dNTP substrate-binding site. All these amino acids residues of Y181-Y188 region held together with the help of peptide bonds (-CONH-). The carbonyl group of amino acids of dNTP substrate binding may involve in the polar interaction with the polar groups on the compounds (ligands). The polar representations of the carbonyl group indicate that the carbon atom will be somewhat positive and the oxygen atom somewhat negative. This suggests two possible modes of reaction for a carbonyl group. The electron deficient (electrophilic) carbon atom can react with nucleophile and the electron rich (nucleophilic) oxygen atom can react with electrophiles. We normally classify the reactions as nucleophilic addition because bond formation to the carbonyl carbon atom by an electron rich reagent is the most significant change that occurs. . It is well established that the stability of the compound formed between nucleophile and electrophile depends upon the value of difference between softness values of $E^{*} \mathrm{~m}$ of nucleophile and softness values of $E^{*} \mathrm{n}$ of electrophile, $\Delta \mathrm{E}^{\star} \mathrm{nm}$ represent the difference. The higher is the $\Delta E^{\ddagger} \mathrm{nm}\left(\Delta E^{\ddagger} \mathrm{nm}=\left|E^{\ddagger} n-E^{\ddagger} m\right|\right)$ greater is the stability of the compound ${ }^{[22-24]}$.

$\Delta \mathrm{E}^{\ddagger} \mathrm{nm}$ values, when the compounds treated as nucleophile and receptor amino acids (Asn-Y182, TyrY183 and Glu-Y186) as electrophile, have shown that interaction occur between the compound $(\mathrm{O} / \mathrm{S}$-atom at site-2) and Asn Y182 amino acid (C-atom of carbonyl group of-CONH-), as the interaction have higher value of $\Delta \mathrm{E}^{\dagger} \mathrm{nm}$ than interaction between Tyr-Y183 and GluY185, 186, Table 9. While in the other case, the compounds (C-atom of site-2) treated as electrophiles and receptor amino acids (O-atom of carbonyl group of$\mathrm{CONH}-)$ as nucleophiles, interaction occurs between the compound (C-atom at site-2) and Asn Y182 amino acid (O-atom of carbonyl group of (-CONH-), as the interaction have higer value of $\Delta \mathrm{E}^{*} \mathrm{~nm}$ than interaction between Tyr-Y183 and Glu-Y186, 186, Table 10. Table 9 and 10 shows that later case has higher values of $\Delta \mathrm{E}^{\ddagger} \mathrm{nm}$ than former and thus the compounds formed between Asn-Y182 amino acid and TIBO have higher stability.

\section{CONCLUSION}

The study has shown that there is a direct relationship between the $\log$ P, SASA and activity of 
the compounds and as $\log \mathrm{P}$ and SASA decreases activity decreases. Thus hydrophobic interaction was predominant and made major contribution, while hydrogen bonding and polar interactions help in proper orientation of the compound (or its functional groups) to make maximam interaction. The overall strengths of these bonds determine the degree of affinity between the drug and the receptor. Thus, the study provide a theoretical way by which new hypothetical HIV-1NNRT inhibitors can be developed prior to their synthesis only by introducing effective hydrophobic substituents at specific sites.

\section{ACKNOWLEDGEMENT}

We thank Dr. Pratibha Singh (Department of Chemistry, M.L.K. (P.G.) College, Balrampur, U.P., India) for valuable suggestions.

\section{REFERENCES}

1. Althaus, I.W., J.J. Chou, A.J. Gonzales, M.R. Deibel, K.C. Chou, F.J. Kezdy, D.L. Romero, R.C. Thomas, P.A. Aristoff, W.G. Tarpley and F. Reusser, 1994. Kinetic-studies with the nonnucleoside humanimmunodeficiency-virus type-1 reversetranscriptase inhibitor u-90152e. Biochem. Pharmacol., 47: 2017-2028. http://www.ncbi.nlm.nih.gov/pubmed/7516658.

2. Jacobo-Molina, A., J. Ding, R.G. Nanni, A.D. Clark, X. Lu, C. Tantillo, R.L. Williams, G. Kamer, A.L. Ferris, P. Clark, A. Hizi, S.H. Hughes and E. Arnold, 1993. Crystal-structure of humanimmunodeficiency-virus type-1 reversetranscriptase complexed with double-stranded DNA at 3.0 angstrom resolution shows bent DNA. Proc. Natl. Acad. Sci. USA., 90: 6320-6324. http://www.ncbi.nlm.nih.gov/pubmed/7687065.

3. Nanni, R.G., J. Ding, M.A. Jacobo, S.H. Hughes and E. Arnold, 1993. Review of HIV-1 reverse transcriptase three-dimensional structure: Implications for drug design. Perspect. Drug Discov. Des., 1: 129-150. DOI: 10.1007/BF02171659.

4. Kohlstaedt, L.A., J. Wang, J.M. Friedman, P.A. Rice and T.A. Steitz, 1992. Crystal-structure at 3.5 angstrom resolution of HIV-1 reverse-transcriptase complexed with an inhibitor. Science, 256: 1783-1790. DOI: $10.1126 /$ science.1377403.

5. De Clercq, E., 1993. HIV-1-specific rt inhibitorshighly selective inhibitors of humanimmunodeficiency-virus type-1 that are specifically targeted at the viral reversetranscriptase. Med. Res. Rev., 13: 229-258. http://www.ncbi.nlm.nih.gov/pubmed/7683360.
6. Pauwels, R., K. andries, Z. Debyser, M.J. Kukla, D. Schols, H.J. Breslin, R. Woestenborghs, J. Desmyter and P.A. Janssen, 1994. New tetrahydroimidazo[4, 5, 1-Jk][1, 4]-benzodiazepin2(1h)-one and tetrahydroimidazo[4, 5, 1-Jk][1, 4]benzodiazepin-2(1h)-thione derivatives are potent inhibitors of human-immunodeficiency-virus type1 replication and are synergistic with 2',3'dideoxynucleoside analogs. Antimicrobial Agents Chemother., 38: 2863-2870. http://www.pubmedcentral.nih.gov/articlerender.fc gi? artid=188298.

7. Debnath, A.K., 2005. Application of 3D-QSAR techniques in anti-HIV-1 drug design-An overview. Curr. Pharm. Des., 11: 3091-3110. http://cat.inist.fr/?aModele=afficheN\&cpsidt=17016029.

8. Horton, H.R., L.A. Moran, R.S. Ochs, J.D. Rawn and K.G. Scrimgeour, 1996. Principles of Biochemistry. 2nd Edn., Prentice Hall, New Jersey, ISBN: 0-13-439167-5, pp: 57-60.

9. Kelly, M.D. and R.L. Mancera, 2005. A new method for estimating the importance of hydrophobic groups in the binding site of a protein. J. Med. Chem., 48: 1069-1078. http://www.ncbi.nlm.nih.gov/pubmed/15715474.

10. Fujita, T., J. Iwasa and C. Hansch, 1964. New substituent constant pi derived from partition coefficients. J. Am. Chem. Soc., 86: 5175.

11. Lee, B. and F.M. Richards, 1971. Interpretation of protein structures-estimation of static accessibility. J. Mol. Biol., 55: 379-400. http://www.ncbi.nlm.nih.gov/sites/entrez?cmd=Ret rieve $\& \mathrm{db}=$ PubMed\&list_uids $=5551392$.

12. Shrake, A. and J.A. Rupley, 1973. Environment and exposure to solvent of protein atoms-lysozyme and insulin. J. Mol. Biol., 79: 351-371. http://www.ncbi.nlm.nih.gov/pubmed/4760134.

13. Horton, H.R., L.A. Moran, R.S. Ochs, J.D. Rawn and K.J. Scrimgeour, 1996. Hydrogen Bond in Water. In: Principles of Biochemistry, 2nd Edn., Prentice Hall, New Jersey, ISBN: 0-13-439167-5, pp: 36.

14. Trapani, G., A. Carotti, M. Franco, A. Latrofa, G. Genchi and G. Liso, 1992. Synthesis and benzodiazepine receptor-binding of some $4 \mathrm{~h}$ pyrimido[2, 1-B]benzothiazol-4-ones. Eur. J. Med. Chem., 27: 39-44. DOI: 10.1016/02235234(92)90058-9.

15. Audry, E., J.P. Dubost, J.C. Colleter and P. Dallet, 1986. A new approach of structure-activityrelationships-the potential of molecular lipophily. Eur. J. Med. Chem., 21: 71-72. http://cat.inist.fr/?aModele $=$ afficheN\&cpsidt $=7869054$. 
16. Gruber, C. and V. Buss, 1989. Quantum mechanically calculated properties for the development of quantitative structure-activityrelationships (qsars)-pka values of phenols and aromatic and aliphatic carboxylic-acids. Chemosphere, 19: 1595-1609. http://cat.inist.fr/?aModele=afficheN\&cpsidt=6757473.

17. Bodor, N., Z. Gabanyi and C.K. Wong, 1989. A new method for the estimation of partitioncoefficient. J. Am. Chem. Soc., 111: 3783-3786. DOI: $10.1021 / \mathrm{ja} 00193 \mathrm{a} 003$.

18. Osmialowski, K., J. Halkiewicz, A. Radecki and R. Kaliszan, 1985. Quantum chemical-parameters in correlation-analysis of gas-liquid chromatographic retention indexes of amines. J. Chromatograph., 346: 53-60.

19. Saura-Calixto, F. and M.A. Garcia-Raso, 1968. J. Chromatogr. Sci., 22: 22.

20. Klopman, G., 1968. Chemical reactivity and concept of charge-and frontier-controlled reactions. J. Am. Chem Soc., 90: 223-234. DOI: 10.1021/ja01004a002.

21. Singh, P.P., S.K.Srivastava and A.K. Srivastava, 1980. Matching between lewis-acids and lewisbases on the basis of quantitative softness values and their relation with the stability of the complexes. 1. J. Inorg. Nucl. Chem., 42: 521-532. DOI: 10.1016/0022-1902(80)80080-7.

22. Singh, P.P., F.A. Pasha and H.K. Srivastava, 2003. DFT based atomic softness and its application in site selectivity. Qsar Combinatorial Sci., 22: 843-851. http://cat.inist.fr/?aModele=afficheN\&cpsidt=15363607.

23. Singh, P.P., F.A. Pasha and H.K. Srivastava, 2003. Noval application of softness parameters for regioselectivity and reaction mechanism. Indian J. Chem. B., 43B: 983-991. http://cat.inist.fr/?aModele $=$ afficheN\&cpsidt $=15740095$.

24. Pasha, F.A., P.P. Singh and H.K. Srivastava, 2005. Semiemperical QSAR study and ligand receptor interaction of estrogens. Mol. Divers., 9: 215-220. DOI: $10.1007 / \mathrm{s} 11030-005-2711-\mathrm{x}$.
25. Dewar, M.J.S. and T.F. Morita, 1969. Ground states of conjugated molecules. 13. Basicities of Conjugated Carbonyl Compounds. J. Am. Chem. Soc., 91: 802-8.6. DOI: 10.1021/ja01032a003.

26. Pearson, R.G., 1989. Absolute Electronegativity and Hardness-Applications to Organic-Chemistry. J. Organ. Chem., 54: 1423-1430. DOI: 10.1021/jo00267a034.

27. Ghose, A.K. and G.M. Crippen, 1986. Atomic physicochemical parameters for 3-dimensional structure-directed quantitative structure-activityrelationships. 1. partition-coefficients as a measure of hydrophobicity. J. Comput. Chem., 7: 565-577. DOI: $10.1002 /$ jcc. 540070419 .

28. Klamt, A. and G. Schuurmann, 1993. Cosmo-a new approach to dielectric screening in solvents with explicit expressions for the screening energy and its gradient. J. Chem. Soc.-Perkin Trans., 2: 799-805. http://cat.inist.fr/?aModele=afficheN\&cpsidt=4780329.

29. Stewart, J.J.P., 1989. Optimization of parameters for semiempirical methods. 1. method. J. Comput. Chem., 10: 209-220. DOI: 10.1002/jcc.540100208.

30. Daniels, D.J., 1996. Surface-penetrating radar. 1st Edn., The Institute of Electrical Engineers, London, ISBN: 10: 0852968620, pp: 300.

31. Ajay, A. and M.A. Murcko, 1995. Computational methods to predict binding free energy in ligandreceptor complexes. J. Med. Chem., 38: 4953-4967. DOI: $10.1021 / \mathrm{jm} 00026 \mathrm{a} 001$.

32. Lum, K., D. Chandler and J.D. Weeks, 1999. Hydrophobicity at small and large length scales. J. Phys. Chem. B., 103: 4570-4577. http://terpconnect.umd.edu/ jdw/jpcb1034570.html.

33. Huang, D.M. and D. Chandler, 2000. Temperature and length scale dependence of hydrophobic effects and their possible implications for protein folding. Proc. Natl. Acad. Sci. USA., 97: 8324-8327. http://www.ncbi.nlm.nih.gov/pubmed/10890881. 